\title{
Monopoles, shockwaves and the classical double copy
}

\author{
Nadia Bahjat-Abbas, Ricardo Stark-Muchão and Chris D. White \\ Centre for Research in String Theory, School of Physics and Astronomy, \\ Queen Mary University of London, \\ 327 Mile End Road, London E1 4NS, U.K. \\ E-mail: n.bahjat-abbas@qmul.ac.uk, r.j.stark-muchao@qmul.ac.uk, \\ christopher.white@qmul.ac.uk
}

ABSTRACT: The classical double copy relates exact solutions in biadjoint scalar, gauge and gravity theories. Recently, nonperturbative solutions have been found in biadjoint theory, which have been speculated to be related to the Wu-Yang monopole in gauge theory. We show that this seems not to be the case, by considering monopole solutions in the infinitely boosted (shockwave) limit. Furthermore, we show that the Wu-Yang monopole is instead related to the Taub-NUT solution, whose previously noted single copy is that of an abelianlike (Dirac) monopole. Our results demonstrate how abelian and non-abelian gauge theory objects can be associated with the same gravity object, and clarify a number of open questions concerning the scope of the classical double copy.

KEywords: Scattering Amplitudes, Solitons Monopoles and Instantons

ARXIV EPRINT: 2001.09918 


\section{Contents}

1 Introduction 1

2 The Kerr-Schild double copy 3

3 Shockwaves and ultraboosts $\quad 7$

3.1 The Aichelburg-Sexl shockwave in Kerr-Schild coordinates 7

$\begin{array}{ll}3.2 \text { Single and zeroth copies } & 10\end{array}$

$\begin{array}{lll}3.3 & \text { Ultraboosting the biadjoint monopole } & 13\end{array}$

4 Relating the Wu-Yang and Dirac monopoles 13

$\begin{array}{llr}5 & \text { Conclusion } & 17\end{array}$

$\begin{array}{lr}\text { A Impulse calculations } & 18\end{array}$

$\begin{array}{ll}\text { A.1 Biadjoint impulse } & 18\end{array}$

$\begin{array}{ll}\text { A.2 Gauge theory impulse } & 19\end{array}$

$\begin{array}{lll}\text { A.3 Gravitational impulse } & 19\end{array}$

B Ultraboosting the general $\mathrm{SU}(2) \times \mathrm{SU}(2)$ monopole $\quad 21$

\section{Introduction}

Field theories continue to be relevant in many different areas of physics. Of particular interest are relativistic quantum theories, needed for particle physics and gravity. Studying the relationships between different theories can be just as important as examining individual theories themselves, given that this may reveal new conceptual insights, or computational methods. One such relationship is the double copy [1-3], whose original incarnation related scattering amplitudes in non-abelian gauge, and gravity, theories - including their supersymmetric generalisations (see refs. [2, 4-46], and ref. [47] for a comprehensive review). This was subsequently extended to classical solutions [48-86], which has a number of applications. Firstly, there is the possibility that the double copy could greatly streamline calculations in classical General Relativity, such as those needed for gravitational wave physics. Secondly, extending the remit of the double copy broadens our conceptual understanding as to whether this is a deep and fundamental connection between different types of field theory, or merely a coincidence for certain observables. If the former is true, it suggests that our traditional way of formulating field theories may be incomplete and / or hiding crucial underlying features.

If the double copy is a complete relationship between gauge and gravity theories, it must somehow be applicable to all possible types of solution. All previous examples 
involving amplitudes or classical solutions (exact or otherwise) involve positive powers of the coupling constants in the relevant theories. It remains to be seen whether or not the double copy can be made truly non-perturbative, i.e. applicable to strong-coupling solutions, containing negative powers of the coupling. As a first step, a recent series of papers has derived such solutions for biadjoint scalar field theory, with the hope of matching them with known strong-coupling solutions in gauge theory [87-89]. One of these represents a monopole-like object, and ref. [87] speculated that this might be relatable to the wellknown Wu-Yang monopole in gauge theory [90]. However, it has remained unclear how to systematically construct such a double copy, whose rules must in any case be fundamentally different to any previous case.

In this paper, we investigate the question of whether the biadjoint monopole of ref. [87] maps to the $\mathrm{Wu}$-Yang monopole, and conclude that it does not. We present two arguments, where the first involves infinitely boosting classical solutions in the various theories we consider, and constructing shockwave solutions from them. In gravity, this was first considered by Aichelburg and Sexl [91], who found that certain parameters have to be rescaled when boosting, in order to keep physically measurable effects finite. We will follow convention by referring to this general procedure as an ultraboost in what follows. Related examples including gravity and / or gauge theory can be found in refs. [92-97]. ${ }^{1}$ Certain shockwave solutions are known to double copy $[32,48,98]$, and thus comparing the ultraboosted biadjoint and $\mathrm{Wu}$-Yang monopole solutions allows us to confirm or refute whether or not they are connected by the double copy. Indeed, we will see that, whilst the Wu-Yang monopole survives its ultraboost, the biadjoint monopole does not, which seems to indicate that they are not after all related. This conclusion is not watertight, however, given that the physics in different theories can turn out to be very different, even if the double copy relates them.

In the second part of our study, we thus seek to explain why we could have expected $a$ priori that the biadjoint and $\mathrm{Wu}$-Yang monopole solutions are not related via the double copy. We recall the existence of a singular gauge transformation that can be used to transform the Wu-Yang solution into a non-abelian version of the Dirac magnetic monopole in electromagnetism, whose form linearises the Yang-Mills equations [99, 100]. The Dirac monopole is known to double copy to the pure NUT solution in gravity. Furthermore, its counterpart in biadjoint theory is already known [49], and does not coincide with the non-perturbative biadjoint monopole of ref. [87]. Thus, there is no room for the nonperturbative biadjoint monopole in matching up shockwaves, which is consistent with it not surviving in the ultraboost limit.

Despite the negative result of our investigation, it proves to be worthwhile for several reasons. Firstly, although both static solutions and shockwaves are known to double-copy, the latter (in gauge and biadjoint theory) have not been explicitly obtained from the former using an ultraboost procedure in this context. The details of how to perform an ultraboost in biadjoint theory are new, and the comparison of this procedure with its gauge and gravity counterparts proves interesting. Secondly, the above-mentioned identification of

\footnotetext{
${ }^{1}$ See also ref. [68] for an intriguing study drawing parallels between gravity and Yang-Mills in the ultraboosted limit.
} 
the $\mathrm{Wu}$-Yang and Dirac monopoles indicates an emerging picture in which both abelianand non-abelian-like objects can double copy to the same gravity solution, which in turn suggests that the classical double copy of ref. [48] (which always concerns abelian-like objects in the gauge theory) is more general than previously thought. Such behaviour has been seen before in the study of amplitudes [27], and is consistent with the fact that colour information is removed when taking the double copy.

The structure of our paper is as follows. In section 2, we review salient details concerning the classical double copy procedure of ref. [48]. In section 3, we show how to obtain shockwaves which have previously been shown to double copy via an ultraboost procedure in biadjoint, gauge and gravity theories. We also find and interpret the ultraboost of the non-perturbative biadjoint monopole of ref. [87]. In section 4, we review a known singular gauge transformation relating the $\mathrm{Wu}$-Yang and Dirac monopoles, and explain the implications of this relationship for the double copy. Finally, in section 5, we discuss our results and conclude.

\section{The Kerr-Schild double copy}

The classical double copy of ref. [48] is a systematic procedure for relating certain exact classical solutions in biadjoint, gauge and gravity theories. In the latter, we may define a graviton field $h_{\mu \nu}$ via

$$
g_{\mu \nu}=\eta_{\mu \nu}-\kappa h_{\mu \nu}, \kappa=\sqrt{16 \pi G_{N}},
$$

with $g_{\mu \nu}\left(\eta_{\mu \nu}\right)$ the full (Minkowski) metric with signature $(+,-,-,-)$, and $G_{N}$ the Newton constant. We may then consider the family of Kerr-Schild solutions, for which the graviton has the special form

$$
h_{\mu \nu}=\frac{\kappa}{2} \phi k_{\mu} k_{\nu}
$$

where $\phi$ is a harmonic function, and the Kerr-Schild vector $k^{\mu}$ is geodesic, and null with respect to either the Minkowski or full metrics:

$$
k \cdot \partial k^{\mu}=0, \quad g_{\mu \nu} k^{\mu} k^{\nu}=\eta_{\mu \nu} k^{\mu} k^{\nu}=0 .
$$

Upon substituting the ansatz of eq. (2.2) into the Einstein equations, they linearise, such that eq. (2.2) represents an exact solution that is particularly tractable. Given the quantities $\phi$ and $k^{\mu}$ appearing in eq. (2.2), one may construct a non-abelian gauge field

$$
\mathbf{A}_{\mu} \equiv A_{\mu}^{a} \mathbf{T}^{a}, \quad A_{\mu}^{a}=c^{a} \phi k_{\mu},
$$

where $\mathbf{T}^{a}$ is a generator of the gauge group with adjoint index $a$, and $c^{a}$ an arbitrary colour vector. Reference [48] proved that for any time-independent Kerr-Schild solution, the gauge field of eq. (2.4) satisifes the linearised Yang-Mills equations, and thus represents an exact physical solution of a non-abelian gauge theory. Note that the procedure for obtaining $A_{\mu}^{a}$ is simply to replace one copy of the Kerr-Schild vector $k_{\mu}$ with the colour vector $c^{a}$ (correspondingly, a spacetime index of the field $h_{\mu \nu}$ is replaced with a colour index in the field $A_{\mu}^{a}$ ). One may repeat this procedure, so as to obtain a field

$$
\mathbf{\Phi}=\Phi^{a a^{\prime}} \mathbf{T}^{a} \tilde{\mathbf{T}}^{a^{\prime}}, \quad \Phi^{a a^{\prime}}=c^{a} \tilde{c}^{a^{\prime}} \phi,
$$




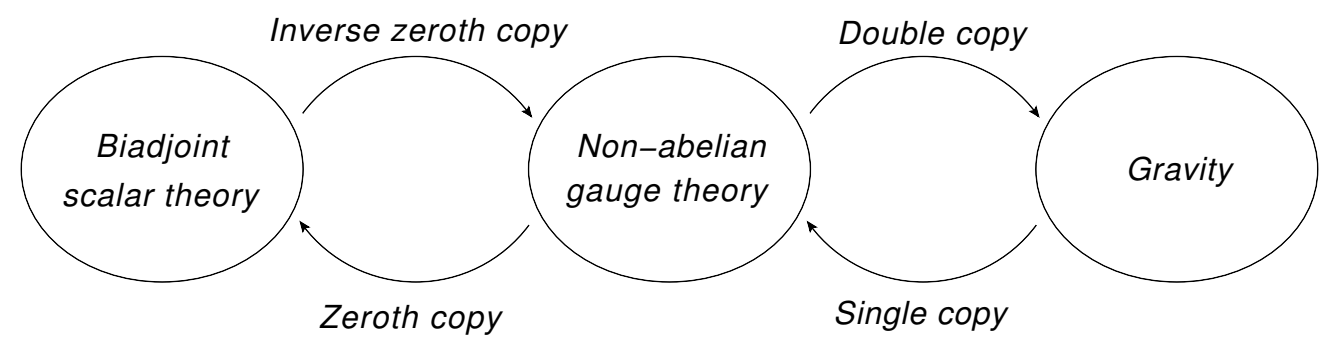

Figure 1. The different theories related by the double, single and zeroth copies.

where $\tilde{c}^{a^{\prime}}$ is a second colour vector, that is potentially associated with a different gauge group to that of $c^{a}$. As shown in ref. [48], eq. (2.5) is a solution of a linearisation of the biadjoint scalar field equation

$$
\partial^{2} \Phi^{a a^{\prime}}-\lambda f^{a b c} \tilde{f}^{a^{\prime} b^{\prime} c^{\prime}} \Phi^{b b^{\prime}} \Phi^{c c^{\prime}}=0
$$

where $\lambda$ is a coupling constant, and $\left\{f^{a b c}\right\},\left\{\tilde{f}^{a^{\prime} b^{\prime} c^{\prime}}\right\}$ are sets of structure constants associated with the two Lie groups. Starting with a time-independent Kerr-Schild solution, we thus obtain solutions in a ladder of different field theories, as depicted in figure 1, where for convenience we also display the name of the map between any given two types of theory. ${ }^{2}$

The above situation mirrors the existence of similar double copy relations between amplitudes in these theories, such that our current understanding is that the classical and amplitude double copies are manifestations of the same underlying correspondence, and indeed overlap where relevant [48-50, 104, 105]. Despite this, a number of open questions exist regarding the classical double copy. Firstly, there is the issue of whether arbitrary sources, as well as the fields themselves, can be furnished with a double-copy interpretation $[50,80,106,107]$. This is an interesting issue, which we will not explore in this paper. Secondly, the family of time-independent Kerr-Schild metrics is clearly very special, and a number of studies have tried to go beyond this. Regarding time dependence, ref. [48] pointed out that certain infinite boosted (shockwave) solutions [91, 108], whose correspondence in gauge and gravity theories was first noted in ref. [98], can also be phrased in terms of the Kerr-Schild double copy. The case of an arbitrarily accelerating point particle was considered in ref. [50], and related to known scattering amplitudes for gluons and gravitons in the Bremsstrahlung limit. Generalisation from time translation invariance to an arbitrary Killing vector was considered in ref. [107]. There have also been attempts to move beyond the simple Kerr-Schild form. Reference [49] considered the Taub-NUT solution in gravity, which has a double Kerr-Schild form, in which the graviton contains two terms of the form of eq. (2.2), involving different harmonic functions and Kerr-Schild vectors. The latter obey certain mutual orthogonality conditions, but this is no longer sufficient to linearise the Einstein equations in general. Remarkably, linearisation still occurs for the special case of Taub-NUT [109], and the single copy is found to be a point-

\footnotetext{
${ }^{2}$ Figure 1 is not the whole story, but forms a subset of an ever-increasing web of theories related by double-copy-like transformations. See e.g. refs. [47, 101-103] for further details.
} 
like dyon, carrying both electric and magnetic charge. ${ }^{3}$ More generally, one may consider the classical double copy of non-Kerr-Schild solutions, but at the price of having to work order-by-order in perturbation theory [51, 61, 63-65]. Thus, Kerr-Schild coordinates are not a fundamental prequisite for being able to construct a classical double copy. Rather, they constitute a maximally convenient case, in which it is possible to make statements to all orders in perturbation theory. ${ }^{4}$

A third puzzle regarding the Kerr-Schild double copy concerns the fact that the single copy of the gravity solution is always abelian-like, in that it linearises the Yang-Mills equations (n.b. the gauge theory solution is still formally non-abelian, in that it is dressed by the colour dependence of eq. (2.4)). Whilst this follows from the mathematical arguments of ref. [48], this situation is slightly at odds with the double copy story for amplitudes, in which the non-abelian nature of the gauge theory plays a pivotal role, through the so-called $B C J$ duality [1] relating colour and kinematic information. It would perhaps be more desirable if one could associate fully non-linear solutions in the gauge theory with gravitational counterparts, and there is also the possibility that both abelian-like and fully non-linear solutions in the gauge theory may map to the same gravity solution. There is in fact a precedent for this in an amplitudes context [27], and we will return to this in what follows.

Before moving on, it is worth providing those examples of the Kerr-Schild double copy that we will later rely on. Arguably the simplest example is that of a pointlike mass sourcing a Schwarzschild black hole, for which the quantities appearing in eq. (2.2) are given by

$$
\phi=\frac{M}{4 \pi r}, \quad k_{\mu}=\left(1, \frac{\boldsymbol{x}}{r}\right),
$$

where $\boldsymbol{x}=(x, y, z)$ is the radial position vector in Cartesian coordinates. The single copy of this is a point charge at the origin, given by

$$
A_{\mu}^{a}=\frac{g c^{a}}{4 \pi r} k_{\mu}
$$

and the zeroth copy is then

$$
\Phi^{a a^{\prime}}=\frac{\lambda c^{a} \tilde{c}^{a^{\prime}}}{4 \pi r} .
$$

Another case we will utilise is that of the Aichelburg-Sexl solution in gravity, representing a shockwave moving along the $x$ direction at the speed of light, and such that the gravitational impulse imparted to a stationary test particle is finite [91]. This has a Kerr-Schild form, with:

$$
\phi=-\frac{M}{4 \pi} \log \left(\frac{\rho}{\rho_{0}}\right) \delta(u), \quad k_{\mu}=(1,-1,0,0),
$$

where

$$
u=t-x
$$

\footnotetext{
${ }^{3}$ Recently, the known electromagnetic duality relating electric and magnetic charges has been explored from a double copy point of view $[84,110,111]$.

${ }^{4} \mathrm{~A}$ related programme of work has shown that a classical double copy is possible in arbitrary coordinate systems, if one restricts to linearised level only [52-56, 59, 60, 112].
} 
is a light-cone coordinate,

$$
\rho=\sqrt{y^{2}+z^{2}}
$$

the cylindrical radius as measured from the $x$ axis, $\rho_{0}$ an arbitrary constant, and the vector $k_{\mu}$ is again expressed in Cartesian coordinates. The single copy of this solution is a non-abelian plane wave [108]

$$
A_{\mu}^{a}=-\frac{g c^{a}}{4 \pi} \log \left(\frac{\rho}{\rho_{0}}\right) \delta(u) k_{\mu}
$$

and there is also a corresponding biadjoint zeroth copy

$$
\Phi^{a a^{\prime}}=-\frac{\lambda c^{a} \tilde{c}^{a^{\prime}}}{4 \pi} \log \left(\frac{\rho}{\rho_{0}}\right) \delta(u) .
$$

In each theory, the factor $\delta(u)$ confines the influence of the field to a plane transverse to the $x$-direction, moving at light speed. There is a non-trival profile function, which depends only on the transverse coordinates $(y, z) .^{5}$

All of the above examples involve positive powers of the coupling constants in the biadjoint, gauge or gravity theories. It remains unknown whether or not the double copy can be extended to non-perturbative solutions, involving inverse powers of the coupling. Solutions of the biadjoint theory play a crucial role in both the amplitude and Kerr-Schild double copies [48]. Thus, it seems natural to assume that this should also be the case in a nonperturbative correspondence, should the latter exist. To this end, refs. [87-89] initiated the programme of cataloguing non-linear solutions of biadjoint theory. The simplest such solution has the form of a static spherically symmetric monopole-like object residing at the origin of spacetime:

$$
\Phi^{a a^{\prime}}=-\frac{2 \delta^{a a^{\prime}}}{\lambda T_{A} r^{2}},
$$

where it is assumed that both Lie groups in the biadjoint theory are the same, and the constant $T_{A}$ is defined in terms of the structure constants via

$$
f^{a b c} f^{a^{\prime} b c}=\delta^{a a^{\prime}} T_{A} .
$$

For the specific case in which the common gauge group is $\mathrm{SU}(2)$, there is also a continuous family of solutions given by

$$
\Phi^{a a^{\prime}}=\frac{1}{\lambda r^{2}}\left[-k\left(\delta^{a a^{\prime}}-\frac{x^{a} x^{a^{\prime}}}{r^{2}}\right) \pm \sqrt{2 k-k^{2}} \frac{\epsilon^{a a^{\prime} d} x^{d}}{r}\right], \quad 0 \leq k \leq 2 .
$$

There was already some speculation in ref. [87] about whether any of these solutions could be related to pointlike objects in non-abelian gauge theory. In the case of $\mathrm{SU}(2)$, a natural candidate is the $W u$-Yang monopole of ref. [90], which in a particular gauge takes the form

$$
A_{0}^{a}=0, \quad A_{i}^{a}=-\frac{\epsilon_{i a k} x^{k}}{g r^{2}},
$$

\footnotetext{
${ }^{5}$ The above shockwave solutions provide a nice link between the classical and amplitude double copies: the gauge and gravity shockwaves were constructed from an all-order Feynman diagram analysis in ref. [32].
} 
where $g$ is the coupling constant. Like the solutions of eq. (2.15), (2.17), this contains an inverse power of the coupling, and also has a pure power-like dependence in the spherical radius $r$, where the power itself can be dictated on dimensional grounds. ${ }^{6}$ Given the lack of any precedent for how to formulate a non-perturbative double copy, ref. [87] left as merely speculative the suggestion that the biadjoint monopoles of eqs. (2.15), (2.17) are related to the $\mathrm{Wu}$-Yang monopole. Our aim here is to examine this systematically, and the starting point will be the above-mentioned fact that shockwave solutions are known to double copy. By ultraboosting the biadjoint monopole, it may turn out to have properties corresponding to a known shockwave, or exhibit other simplifying features that enable a suitable double copy interpretation to be obtained. If it instead does not survive the ultraboost, then this is evidence that the speculative link between the biadjoint and $\mathrm{Wu}$-Yang monopoles may in fact be incorrect.

\section{Shockwaves and ultraboosts}

Above, we have suggested ultraboosting monopole solutions in biadjoint and gauge theories, in order to see what can be learned about the possible existence of a nonperturbative double copy. Before considering the case of the biadjoint monopole, however, it pays to revisit the Aichelburg-Sexl family of shockwaves, written here in eqs. (2.10), (2.13), (2.14). We will recast the ultraboost procedure of ref. [91] using Kerr-Schild coordinates, so that double copy properties are manifest. This will also allow us to examine the ultraboost in the biadjoint scalar theory, which has not been previously considered.

\subsection{The Aichelburg-Sexl shockwave in Kerr-Schild coordinates}

Let us begin with the point mass (Schwarzschild) solution of eqs. (2.2), (2.7), taken to be stationary in an inertial frame $S^{\prime}$ with Cartesian coordinates $\left(t^{\prime}, x^{\prime}, y^{\prime}, z^{\prime}\right)$. We may then consider that $S^{\prime}$ is moving with boost parameter $\beta \equiv v$ (in natural units) in the $+x$ direction relative to a second frame $S$ whose coordinates are $(t, x, y, z)$. The two sets of coordinates are related by the Lorentz transformation

$$
\left(\begin{array}{l}
t^{\prime} \\
x^{\prime} \\
y^{\prime} \\
z^{\prime}
\end{array}\right)=\left(\begin{array}{cccc}
\gamma & -\gamma \beta & 0 & 0 \\
-\gamma \beta & \gamma & 0 & 0 \\
0 & 0 & 1 & 0 \\
0 & 0 & 0 & 1
\end{array}\right)\left(\begin{array}{l}
t \\
x \\
y \\
z
\end{array}\right)=\left(\begin{array}{c}
\gamma(t-\beta x) \\
\gamma(x-\beta t) \\
y \\
z
\end{array}\right)
$$

The graviton in $S^{\prime}$ is given in Kerr-Schild form by

$$
h_{\mu \nu}^{\prime}=\frac{\kappa}{2} \phi\left(x^{\prime}\right) k_{\mu}^{\prime} k_{\nu}^{\prime}
$$

\footnotetext{
${ }^{6}$ Note that $g$ in the gauge theory is dimensionless in four spacetime dimensions, whereas the coupling constant $\lambda$ in eq. (2.6) has dimensions of mass.
} 
where the function $\phi$ and Kerr-Schild vector are given in terms of the primed coordinates by eq. (2.7). Boosting these ingredients to the unprimed frame, one finds

$$
\begin{aligned}
\phi(x)= & \frac{M}{4 \pi} \frac{1}{\left[\gamma^{2}(x-\beta t)^{2}+\rho^{2}\right]^{1 / 2}}, \\
k_{\mu}= & \left(\gamma-\frac{\gamma^{2} \beta(x-\beta t)}{\left[\gamma^{2}(x-\beta t)^{2}+\rho^{2}\right]^{1 / 2}},-\gamma \beta+\frac{\gamma^{2}(x-\beta t)}{\left[\gamma^{2}(x-\beta t)^{2}+\rho^{2}\right]^{1 / 2}}, \frac{y}{\left[\gamma^{2}(x-\beta t)^{2}+\rho^{2}\right]^{1 / 2}},\right. \\
& \left.\frac{z}{\left[\gamma^{2}(x-\beta t)^{2}+\rho^{2}\right]^{1 / 2}}\right) .
\end{aligned}
$$

To look for a shockwave solution, we must take the limit $\gamma \rightarrow \infty$, whilst also regularising the solution so that physically measurable quantities are finite. One such quantity is the deflection of a test particle upon crossing the shockwave, which is linear in the field. Thus, we require that $h_{\mu \nu}$ is finite in the ultraboost limit. However, the limiting procedure itself is rather subtle, given that one finds different results for the limiting values of the quantities in eq. (3.2) depending upon whether one is inside $(x=\beta t)$ or outside $(x \neq \beta t)$ the plane of the shockwave. For the former case one obtains

$$
\phi \stackrel{\gamma \rightarrow \infty}{\longrightarrow} \frac{M}{4 \pi} \frac{1}{\rho}+\mathcal{O}\left(\gamma^{-1}\right), \quad k_{\mu} \stackrel{\gamma \rightarrow \infty}{\longrightarrow} \gamma \bar{k}_{\mu}+\mathcal{O}\left(\gamma^{0}\right), \quad x=\beta t,
$$

where we have defined the dimensionless 4 -vector

$$
\bar{k}_{\mu}=(1,-1,0,0) .
$$

Outside the shockwave plane one obtains

$$
\phi \stackrel{\gamma \rightarrow \infty}{\longrightarrow} \frac{M}{4 \pi} \frac{1}{\gamma|t-x|}+\mathcal{O}\left(\gamma^{-2}\right), \quad k_{\mu} \stackrel{\gamma \rightarrow \infty}{\longrightarrow} 2 \gamma \theta(t-x) \bar{k}_{\mu}+\mathcal{O}\left(\gamma^{0}\right), \quad x \neq \beta t,
$$

where $\theta(t-x)$ is the Heaviside function. The complete boosted graviton field is then given by

$$
h_{\mu \nu} \stackrel{\gamma \rightarrow \infty}{\longrightarrow} \frac{\kappa}{2} \frac{M}{4 \pi} \begin{cases}\frac{\gamma^{2}}{\rho} \bar{k}_{\mu} \bar{k}_{\nu}+\mathcal{O}(\gamma), & x=\beta t \\ \frac{4 \gamma}{|t-x|} \theta(t-x) \bar{k}_{\mu} \bar{k}_{\nu}+\mathcal{O}\left(\gamma^{0}\right), & x \neq \beta t\end{cases}
$$

It is clear that things are badly divergent, both on and off the plane, which makes physical sense: boosting a massive particle to light speed requires infinite energy, and thus will result in a divergent field configuration! The transition to a finite shockwave solution proceeds as follows [91]. First, one may rescale the $\operatorname{mass}^{7}$ according to

$$
M \rightarrow \frac{M}{\gamma}
$$

Then the graviton field of eq. (3.6) remains infinite inside the plane $x=\beta$, but not outside it. This suggests that the ultraboosted field could indeed contain a delta function

\footnotetext{
${ }^{7}$ In the literature this rescaling is noted to keep the energy finite whilst taking the rest mass to zero. Physically one may view this as a necessary step in changing description between a massive and a massless particle.
} 
$\delta(u)$, localising the extent of the field to the shockwave plane only. To recognise the delta function, we may reinstate the $\gamma$-dependence for the field near the shockwave plane, writing this as

$$
h_{\mu \nu} \stackrel{\gamma \rightarrow \infty, M \rightarrow M / \gamma}{\longrightarrow} \frac{\kappa}{2} \frac{\gamma M}{4 \pi} \frac{1}{\left[\gamma^{2}(x-\beta t)^{2}+\rho^{2}\right]^{1 / 2}} \bar{k}_{\mu} \bar{k}_{\nu} .
$$

We may then attempt to use the general formula (see e.g. ref. [97])

$$
\lim _{\gamma \rightarrow \infty} \gamma f(\gamma u)=\delta(u) \int_{-\infty}^{\infty} d w f(w)
$$

for expressing a Dirac delta function as the limit of a delta sequence $f(u)$, namely a function that can be continuously transformed to make an infinitely sharp peak at $u=0$. In the present case, we may identify

$$
f(u)=\frac{M}{4 \pi} \frac{1}{\left(u^{2}+\rho^{2}\right)^{1 / 2}},
$$

such that eq. (3.9) indeed corresponds to taking the limit of the prefactor in eq. (3.8). However, application of eq. (3.9) then fails due to the fact that the integral on the righthand side is not convergent. We will interpret the physics of this shortly, but for now note that we can modify the function $f(u)$ according to ${ }^{8}$

$$
f(u) \rightarrow \frac{M}{4 \pi}\left[\frac{1}{\left(u^{2}+\rho^{2}\right)^{1 / 2}}-\frac{1}{\left(u^{2}+\rho_{0}^{2}\right)^{1 / 2}}\right]
$$

such that the integral on the right-hand side of eq. (3.9) becomes

$$
\begin{aligned}
\int_{-\infty}^{\infty} d u f(u) & =\frac{M}{4 \pi} \lim _{\epsilon \rightarrow 0} \int_{-\infty}^{\infty} d u\left[\left(u^{2}+\rho^{2}\right)^{-1 / 2+\epsilon}-\left(u^{2}+\rho_{0}^{2}\right)^{-1 / 2+\epsilon}\right] \\
& =\frac{M}{4 \pi} \lim _{\epsilon \rightarrow 0}\left\{\frac{\sqrt{\pi} \Gamma(-\epsilon)}{\Gamma(1 / 2-\epsilon)}\left[\left(\rho^{2}\right)^{\epsilon}-\left(\rho_{0}^{2}\right)^{\epsilon}\right]\right\},
\end{aligned}
$$

where we have chosen to introduce a regularisation parameter $\epsilon$. The latter reveals that each of the separate terms in eq. (3.12) is divergent, but that the combination produces the well-defined limit

$$
\int_{-\infty}^{\infty} d u f(u)=-\frac{M}{4 \pi} \log \left(\frac{\rho^{2}}{\rho_{0}^{2}}\right)
$$

The complete form for the ultraboosted field is now

$$
h_{\mu \nu} \stackrel{\gamma \rightarrow \infty, M \rightarrow M / \gamma}{\longrightarrow}-\frac{\kappa}{2} \frac{M}{4 \pi} \log \left(\frac{\rho}{\rho_{0}}\right) \delta(u) \bar{k}_{\mu} \bar{k}_{\nu}
$$

which is precisely the Aichelburg-Sexl shockwave of eq. (2.10). We can now interpret the divergence that appeared in trying to apply eq. (3.9) to the original function of eq. (3.10): its regularisation merely amounts to introducing an overall constant, that sets the scale of the logarithmic prefactor in eq. (3.14), and which has to be present on dimensional grounds.

\footnotetext{
${ }^{8}$ Reference [91] justifies this transformation based on the fact that it corresponds to a diffeomorphism of the graviton field.
} 
It has no physical consequences, given that deflections of test particles depend only upon derivatives of the metric.

Note that in Kerr-Schild coordinates, the combination of the mass rescaling and regularisation procedures can be rephrased in a particularly simple form. The correct ultraboost of the Schwarzschild solution to form an Aichelburg-Sexl shockwave consists of the following two steps:

(i) One must rescale the mass parameter according to eq. (3.7).

(ii) One must modify the boosted Kerr-Schild function in eq. (3.2) according to

$$
\phi(x) \rightarrow \frac{M}{4 \pi}\left[\frac{1}{\left[\gamma^{2}(x-\beta t)^{2}+\rho^{2}\right]^{1 / 2}}-\frac{1}{\left[\gamma^{2}(x-\beta t)^{2}+\rho_{0}^{2}\right]^{1 / 2}}\right] .
$$

This is slightly different to the above analysis, in that one modifies the $\phi$ function before taking the limit $\gamma \rightarrow \infty$. Importantly, this modification maintains the fact that $\phi$ is harmonic, so that the Kerr-Schild conditions still apply. Repeating the above analysis yields eq. (3.14) as before. ${ }^{9}$ By modifying $\phi$ itself, however, we have cast the ultraboost procedure into a form which has a natural counterpart under the single and zeroth copies.

\subsection{Single and zeroth copies}

In the previous section, we have given an explicit procedure for ultraboosting a point mass in Kerr-Schild coordinates. We may carry out a similar procedure for the point charge in a gauge theory, for which one may start with the single copy of the Schwarzschild solution in the primed coordinate system, and boost it to obtain the following gauge field:

$$
\mathbf{A}_{\mu}=\phi k_{\mu} .
$$

Here $\phi$ is given by the function of eq. (3.2), but with the mass replaced by the colour charge $c^{a} \mathbf{T}^{a}$, and $k_{\mu}$ is also given in eq. (3.2). For the ultraboost, we may again modify $\phi$ according to eq. (3.15), after which we find the following limit:

$$
\mathbf{A}_{\mu} \stackrel{\gamma \rightarrow \infty}{\longrightarrow}-\frac{c^{a} \mathbf{T}^{a}}{4 \pi} \log \left(\frac{\rho}{\rho_{0}}\right) \delta(u) \bar{k}_{\mu}
$$

where $\bar{k}_{\mu}$ has been defined in eq. (3.4). This is precisely the form of the gauge theory shockwaves considered in refs. [32, 108] (see also ref. [96] for an interesting discussion of gauge theory shockwaves in a different context). Note that to obtain a finite field configuration, there is no need to rescale the charge, as was needed for the mass parameter in the gravity theory. We will return to this point below.

For the zeroth copy, we may simply consider the biadjoint field (in the primed coordinate system)

$$
\Phi^{a a^{\prime}}=\frac{\lambda c^{a} \tilde{c}^{a^{\prime}}}{4 \pi r^{\prime}} .
$$

\footnotetext{
${ }^{9} \mathrm{An}$ advantage of this alternative way of formulating the ultraboost is that the boosted field manifestly vanishes off of the shockwave plane, in contrast to eq. (3.6). Indeed, the latter behaviour is associated with the fact that the original $\phi$ function could not be used to form a delta sequence.
} 


\begin{tabular}{|c|c|c|}
\hline Theory & Parameter & Rescaling \\
\hline Biadjoint scalar & $c^{a} \tilde{c}^{a^{\prime}}$ & $\gamma$ \\
Gauge theory & $c^{a}$ & 1 \\
Gravity & $M$ & $\gamma^{-1}$ \\
\hline
\end{tabular}

Table 1. Different scalings needed for the source parameters in different theories, where the factor in the third column multiplies the parameter in question.

Boosting to the unprimed system and making the modification of eq. (3.15), one obtains the limit

$$
\Phi^{a a^{\prime}} \stackrel{\gamma \rightarrow \infty, c^{a} \tilde{c}^{a^{\prime}} \rightarrow \gamma c^{a} \tilde{c}^{a^{\prime}}}{\longrightarrow}-\frac{\lambda c^{a} \tilde{c}^{a^{\prime}}}{4 \pi} \log \left(\frac{\rho}{\rho_{0}}\right) \delta(u),
$$

where in order to achieve a non-vanishing field configuration, we must rescale the charges as shown. Upon doing so, the shockwave solutions of eqs. (3.14), (3.17), (3.19) are related directly by the usual single and zeroth copy procedures. That is, one may write eq. (3.14) in the form

$$
h_{\mu \nu}=\frac{\kappa}{2} \bar{\phi} \bar{k}_{\mu} \bar{k}_{\nu}, \quad \bar{\phi}=-\frac{M}{4 \pi} \log \left(\frac{\rho}{\rho_{0}}\right) \delta(u),
$$

where $\bar{k}_{\mu}$ (from eq. (3.4)) is indeed null and geodesic, and $\bar{\phi}$ harmonic. The single and zeroth copies imply that one must remove factors of $\bar{k}_{\mu}$, and replace mass with charge accordingly, leading directly to eqs. (3.17) and (3.19). We can also make sense, from a double copy point of view, of the modification of eq. (3.15). As stressed in ref. [48], the function $\phi$ in eq. (2.2) can be interpreted as a scalar propagator, and is analogous to the denominator factors in amplitudes, which are not modified upon taking the double copy from gauge theory to gravity. In the present case, the function $\bar{\phi}$ that we arrive at after the ultraboost is indeed the known propagator in two spatial dimensions (i.e. corresponding to the transverse plane), and the modification of eq. (3.15) is necessary so as to construct the most general form of the propagator by including the constant $\rho_{0}$. This in turn explains why eq. (3.15) is necessary when performing ultraboosts in all three theories of figure 1.

Above, we have seen that different scalings of mass / charge parameters in different theories are necessary, to obtain a finite field. The sources for the biadjoint, gauge and gravity theories respectively are as follows:

$$
\rho^{a a^{\prime}}=c^{a} \tilde{c}^{a^{\prime}} \delta^{(3)}(\boldsymbol{x}), \quad j_{\mu}^{a}=c^{a} \delta_{\mu}^{0} \delta^{(3)}(\boldsymbol{x}), \quad T_{\mu \nu}=M \delta_{\mu}^{0} \delta_{\nu}^{0} \delta^{(3)}(\boldsymbol{x}) .
$$

The rescalings act on the parameters entering these source terms, and we summarise the different findings in table 1. Confirmation that these are indeed the correct scalings to obtain finite physical effects can be verified by placing a test particle away from the origin ${ }^{10}$ in the plane $x=0$, and calculating the impulse

$$
\delta p_{\mu}=\int_{-\infty}^{\infty} d t \frac{d p_{\mu}}{d t}
$$

\footnotetext{
${ }^{10}$ The shockwave profile diverges at $y=z=0$, so that we must place the test particle elsewhere.
} 
that it receives as the shockwave passes. In biadjoint theory the integrand is given by [61]

$$
\frac{d p_{\mu}}{d t}=-\frac{\lambda}{m} c_{2}^{a} \tilde{c}_{2}^{a^{\prime}} \partial_{\mu} \Phi^{a a^{\prime}}
$$

where $c_{2}^{a}$ and $\tilde{c}_{2}^{a^{\prime}}$ are the colour charge vectors associated with the test particle. Substituting eq. (3.19) into eq. (3.23) and integrating according to eq. (3.22), one finds (see appendix A for more details)

$$
\delta p_{\text {scal. }}^{\mu}=-\frac{\lambda^{2}}{m} \frac{c \cdot c_{2} \tilde{c} \cdot \tilde{c}_{2}}{2 \pi \rho}(0,0, \hat{\rho}),
$$

where $\hat{\rho}$ is a unit vector in the radial direction in the plane $x=0$. This is indeed finite as required. Furthermore, it does not depend on the constant $\rho_{0}$, justifying the remarks made above. For the gauge theory, one may use the Lorentz force law

$$
\frac{d p_{\mu}}{d t}=g c_{2}^{a} F_{\mu \nu}^{a} v^{\nu}
$$

where $F_{\mu \nu}^{a}$ is the field strength tensor of the shockwave, $c_{2}^{a}$ the colour charge vector of the test particle, whose initial velocity is $v_{\mu}=(1, \mathbf{0})$. One then finds an impulse

$$
\delta p_{\text {gauge }}^{\mu}=g^{2} \frac{c_{2} \cdot c}{2 \pi \rho}(0,0, \hat{\rho}) .
$$

Finally, for gravity one may use the geodesic equation

$$
\frac{d p^{\mu}}{d t}=-m \Gamma_{\nu \sigma}^{\mu} v^{\nu} v^{\sigma}
$$

where $\Gamma_{\nu \lambda}^{\mu}$ is the Christoffel symbol. One subsequently obtains the impulse

$$
\delta p_{\text {grav. }}^{\mu}=-\frac{\kappa^{2} M m}{8 \pi \rho}(0,0, \hat{\rho}) .
$$

In all cases, the impulses are finite, which indeed is entirely consistent with the fact that the rescalings of table 1 were such as to make the profiles of the fields in the shockwave plane finite: the three force laws of eqs. (3.23), (3.25), (3.27) are all linear in their respective fields. ${ }^{11}$ Turning this around, we can use physical finiteness of the impulses to dictate the rescaling needed in each theory, and the power of $\gamma$ needed to rescale the parameters of the field can then simply be traced to the number of factors of the Kerr-Schild vector each field contains, and hence its spin.

In this section, we have shown how the ultraboost procedure can be implemented in biadjoint, gauge and gravity theories, so as to be fully compliant with the double copy. We focused on point charges or masses that are solutions of the linearised field equations in each case (up to a source term localised at the origin). In all cases, the charges survived the ultraboost, resulting in a well-defined shockwave solution that could be meaningfully mapped between theories as in figure 1. Armed with this experience, let us now see what happens if we try to ultraboost the non-perturbative biadjoint monopole.

\footnotetext{
${ }^{11}$ It is worth pointing out that different physical quantities may require different scalings upon performing an ultraboost, such as the non-linear electromagnetic interactions considered in ref. [96].
} 


\subsection{Ultraboosting the biadjoint monopole}

Starting with the solution of eq. (2.15) in the rest frame $S^{\prime}$, we may boost to the unprimed frame to obtain

$$
\Phi^{a a^{\prime}}=-\frac{2 \delta^{a a^{\prime}}}{\lambda T_{A}} \frac{1}{\left[\gamma^{2}(x-\beta t)^{2}+\rho^{2}\right]} .
$$

Taking the ultraboost limit, one finds

$$
\Phi^{a a^{\prime}} \stackrel{\gamma \rightarrow \infty}{\longrightarrow}-\frac{2 \delta^{a a^{\prime}}}{\lambda T_{A}} \begin{cases}\frac{1}{\rho^{2}}, & x=\beta t \\ \mathcal{O}\left(\gamma^{-2}\right), & x \neq \beta t .\end{cases}
$$

Due to the different dependence on the radial coordinate, this does not diverge on the transverse plane, in contrast to the boosted point charge considered in the previous section. Without this divergence, eq. (3.30) does not constitute a shockwave: it is at most a tepid ripple. That is, it imparts no finite impulse to a test particle, given that a finite field is confined to an infinitely thin plane.

It is interesting to note that one could still obtain a shockwave by rescaling the coupling $\lambda$ in eq. (3.30). Carrying out a similar analysis to the previous section, one may show that

$$
\Phi^{a a^{\prime}} \stackrel{\gamma \rightarrow \infty, \lambda \rightarrow \gamma^{-1} \lambda}{\longrightarrow}-\frac{2 \delta^{a a^{\prime}}}{\lambda T_{A}} \frac{1}{\rho} \delta(u),
$$

which does indeed exert a finite impulse. However, the nature of this rescaling is very different to that of the boosted point charges considered earlier. In the latter cases, the parameters entering the source terms (i.e. masses and charges) were rescaled. For the non-perturbative monopole, there is no such source, which makes it questionable that one should be able to rescale the coupling. ${ }^{12}$

Based on the above considerations, we conservatively conclude that the nonperturbative biadjoint monopole does not survive an ultraboost. Similar considerations are reached for the more general monopoles of eq. (2.17), as we show in appendix B. There are then two possibilities as regards a potential non-perturbative double copy, and in particular the suggestion that the biadjoint monopole could be related to a Wu-Yang monopole in gauge theory. The first is that the two objects are indeed related, but that the physics of ultraboosting is potentially very different in the two theories, such that the biadjoint monopole disappears. Whether or not the Wu-Yang monopole survives an ultraboost is irrelevant for the argument. The second possibility is that the biadjoint monopole disappears because it does not need to match up with a known shockwave solution, and in particular is not related to the $\mathrm{Wu}$-Yang monopole. In the next section, we will explain why it is in fact this second possibility that appears to be correct.

\section{Relating the Wu-Yang and Dirac monopoles}

Above, we saw that the failure of the biadjoint monopole to survive its ultraboost is possible evidence for its not being related to the Wu-Yang monopole after all, contrary to the

\footnotetext{
${ }^{12}$ Coupling constants are of course not constant when quantum corrections are included, leading to renormalisation. But that is not what is happening here.
} 
speculation of ref. [87]. We now explain why this must be the case, and our explanation will itself provide new insights into the remit of the classical double copy itself.

First, we recall that there is a gauge transformation that relates the $\mathrm{Wu}$-Yang monopole in $\mathrm{SU}(2)$ gauge theory to a non-abelian embedding of the Dirac monopole, as noted in refs. $[99,100] .{ }^{13}$ By the latter, we mean a gauge field of the form

$$
A_{\mu}^{a}=c^{a} A_{\mu}^{\text {Dirac }}
$$

where $c^{a}$ is a constant colour vector as usual, and

$$
A_{\mu}^{\text {Dirac }}=\left(0,-\frac{\tilde{g} y}{r(r+z)}, \frac{\tilde{g} x}{r(r+z)}, 0\right)
$$

is a solution of the Maxwell equations corresponding to a Dirac (magnetic) monopole of charge $\tilde{g}$. This has a well-known string singularity, which we have chosen to extend from the origin along the $-z$ direction. Note that eq. (4.2) is reminiscent of the single copy solutions in the Kerr-Schild double copy, in that it is manifestly of a form which linearises the Yang-Mills equations. We may thus refer to this solution as abelian-like, even though it is not, strictly speaking, a solution of an abelian gauge theory.

Without loss of generality for the following arguments, we may choose the constant colour vector to lie in the 3 -direction in the internal space, so that $c^{a}=\delta^{a 3}$. Given that we are focusing on an $\mathrm{SU}(2)$ gauge group, we may thus write the complete (matrix-valued) gauge field as

$$
\mathbf{A}_{\mu}=A_{\mu}^{\text {Dirac }} \sigma_{3}
$$

where

$$
\sigma_{i}=\frac{1}{2} \tau_{i}
$$

is a generator of $\mathrm{SU}(2)$, in terms of the Pauli matrices

$$
\tau_{1}=\left(\begin{array}{ll}
0 & 1 \\
1 & 0
\end{array}\right), \quad \tau_{2}=\left(\begin{array}{rr}
0 & -i \\
i & 0
\end{array}\right), \quad \tau_{3}=\left(\begin{array}{rr}
1 & 0 \\
0 & -1
\end{array}\right) .
$$

We may transform eq. (4.2) to spherical polar coordinates $(t, r, \theta, \phi)$, yielding

$$
\mathbf{A}_{\mu}=\sigma_{3}\left(0,0,0, \frac{\tilde{g}(1-\cos \theta)}{r \sin \theta}\right)_{\text {spherical }} .
$$

Next, we can make a gauge transformation

$$
\mathbf{A}_{\mu} \rightarrow \mathbf{A}_{\mu}^{\prime}=\mathbf{U} \mathbf{A}_{\mu} \mathbf{U}^{-1}+\frac{i}{g} \mathbf{U}\left(\partial_{\mu} \mathbf{U}^{-1}\right)
$$

Here $g$ is the electric charge in the gauge theory, which is related to the magnetic charge by the quantisation condition ${ }^{14}$

$$
g \sim \frac{1}{\tilde{g}}
$$

\footnotetext{
${ }^{13} \mathrm{~A}$ similar gauge transformation is used to relate different forms of the 't Hooft-Polyakov monopole of refs. [113, 114].

${ }^{14}$ Equation (4.8) is defined up to an overall constant, which is fixed by stability of the monopole [99], and irrelevant for the present argument.
} 
Choosing the specific transformation matrix ${ }^{15}$

$$
\mathbf{U}=e^{i \phi \sigma_{3}} e^{i \theta \sigma_{2}} e^{-i \phi \sigma_{3}},
$$

one finds that the transformed field in spherical polar coordinates is given by

$$
\mathbf{A}_{\mu}^{\prime}=\left(0,0, \frac{\tilde{g}}{r}\left(\sin \phi \sigma_{1}-\cos \phi \sigma_{2}\right), \frac{\tilde{g}}{r}\left(\cos \theta \cos \phi \sigma_{1}+\cos \theta \sin \phi \sigma_{2}-\sin \theta \sigma_{3}\right)\right)_{\text {spherical }} .
$$

Finally, transforming back to Cartesian coordinates, one finds

$$
A_{0}^{\prime a}=0, \quad A_{i}^{\prime a}=-\frac{\tilde{g} \epsilon_{i a k} x_{k}}{r^{2}},
$$

such that using eq. (4.8) returns precisely the Wu-Yang form of eq. (2.18). This is distinctly different from the abelian-like form of eq. (4.1): the Levi-Cevita symbol mixes the spatial and gauge indices. In addition, the form of eq. (4.11) no longer linearises the Yang-Mills equations, thus is genuinely non-abelian in this gauge. ${ }^{16}$

This result immediately explains why the analysis of section 3 failed to find conclusive evidence that the ultraboosted biadjoint monopole could be potentially matched with a Wu-Yang monopole: the Wu-Yang monopole is nothing other than the abelian-like Dirac monopole in disguise. In the Kerr-Schild double copy, the latter is known to be related to the NUT solution in gravity [49]. The ultraboosted Dirac monopole then double copies to a so-called NUT wave [97]. We may confirm this explicitly, by taking the gauge field of eq. (4.3) to be static in the inertial frame $S^{\prime}$ of section 3 , and boosting to the frame $S$ of eq. (3.1). The result is

$$
A_{\mu}^{a}=\frac{\tilde{g} \delta^{a 3}}{\left[\gamma^{2}(x-\beta t)^{2}+\rho^{2}\right]^{1 / 2}\left\{\left[\gamma^{2}(x-\beta t)^{2}+\rho^{2}\right]^{1 / 2}+z\right\}}\left(\begin{array}{c}
\gamma \beta y \\
-\gamma y \\
\gamma(x-\beta t) \\
0
\end{array}\right) .
$$

Taking the limit $\gamma \rightarrow \infty$ and using eq. (3.9), one finds

$$
A_{\mu}^{a}=\delta^{a 3} \phi_{M} \bar{k}_{\mu}
$$

where $\bar{k}_{\mu}$ has been given in eq. (3.4), and

$$
\phi_{M}=\tilde{g}\left[\pi-2 \tan ^{-1}\left(\frac{z}{y}\right)\right] \delta(u) .
$$

This has the form of a delta function in the lightcone coordinate $u$, dressed by a profile function $\phi_{M}$ whose dependence is only on the coordinates in the transverse plane, thus is indeed a shockwave. Note that the function $\phi_{M}$ has a cut in the $(y, z)$ plane, that is a

\footnotetext{
${ }^{15}$ Our presentation differs from that of ref. [99] due to our choice of Hermitian, rather than anti-Hermitian, generators.

${ }^{16}$ The fact that one can gauge away the non-abelian nature of the monopole may be related to its static nature [115].
} 


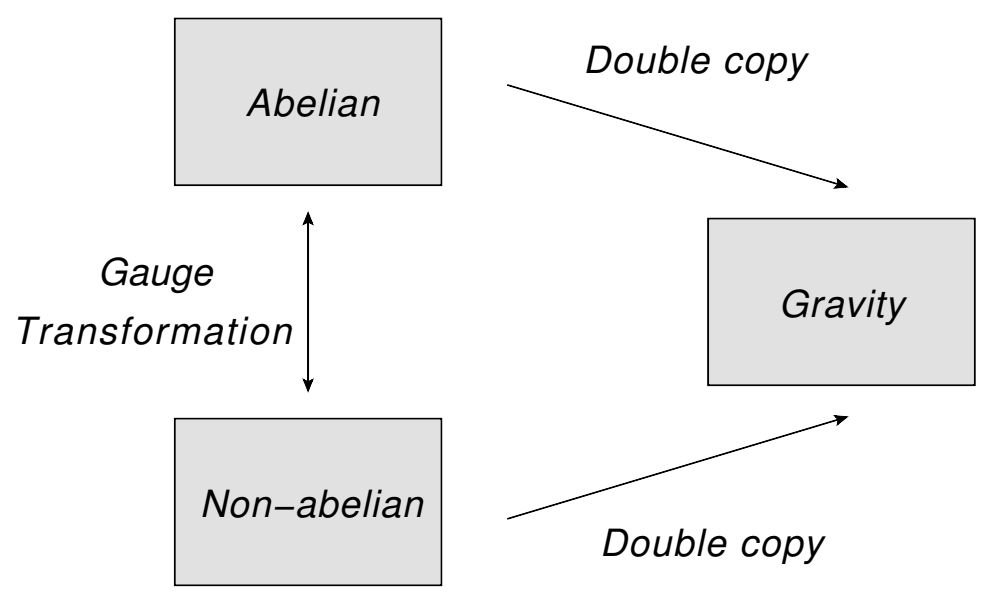

Figure 2. Generalisation of the Kerr-Schild double copy, in which one may identify abelian or non-abelian exact solutions of a gauge theory with the same gravity solution.

remnant of the original Dirac string. As for the latter, we may choose where to place this cut in the shockwave plane, by performing gauge transformations. This would affect the constant term in eq. (4.14), which is therefore not physical by itself.

We may also note that the function $\phi_{M}$ is harmonic, and that $\bar{k}_{\mu}$ (as has already been pointed out above) is null and geodesic. Following the rules of ref. [48], we are thus entitled to take the double copy of eq. (4.13) to obtain the graviton

$$
h_{\mu \nu}=N\left[\pi-2 \tan ^{-1}\left(\frac{z}{y}\right)\right] \delta(u) \bar{k}_{\mu} \bar{k}_{\nu},
$$

where we have replace the magnetic monopole charge $\tilde{g}$ with a gravitational charge $N$. Upon identifying the latter with NUT charge, eq. (4.15) agrees with the NUT wave solution first derived in ref. [97]. ${ }^{17}$ We may also take the zeroth copy of eq. (4.13), to obtain a biadjoint scalar field

$$
\Phi^{a a^{\prime}}=\lambda c^{a} \tilde{c}^{a^{\prime}}\left[\pi-2 \tan ^{-1}\left(\frac{z}{y}\right)\right] \delta(u) .
$$

This is clearly non-zero, and thus not at all identifiable with the ultraboost of the nonperturbative monopole of eq. (2.15), which effectively vanished!

The results of this section are of interest in extending the remit of the classical double copy. A frequently encountered question amongst newcomers to the subject is why the KerrSchild double copy should always produce an abelian-like solution, when the amplitude double copy crucially relies on the fact that the gauge theory is non-abelian (i.e. through BCJ duality [1]). The natural interpretation of the above results, however, is that one may identify the NUT solution in gravity as a double copy either of the abelian-like magnetic monopole of eq. (4.3), or the fully non-abelian Wu-Yang monopole of eq. (2.18). That this is possible is due to the fact that colour information is stripped off when taking the double copy, and the overall scheme is as depicted in figure 2. Interestingly, a similar

\footnotetext{
${ }^{17}$ In order to compare with ref. [97], one must change the boost direction to the $+z$ direction, and also move the position of the cut of the function $\phi_{M}$ in the transverse plane.
} 
picture already has a precedent in the study of scattering amplitudes: ref. [27] examined the infrared singularities of amplitudes in either QED or QCD, and showed that these both matched up with the same structure of IR singularities in GR, to all orders in perturbation theory. Furthermore, it is certainly the case that non-abelian classical solutions can be constructed perturbatively, and double copied [51,61, 63]. However, we believe that our results constitute the first example of an exact non-abelian solution that can be double copied to a gravitational counterpart, and the extension of this to other gauge groups and solutions deserves further investigation.

\section{Conclusion}

In this paper, we have systematically investigated the possibility of a non-perturbative double copy between gauge and gravity theories. More specifically, we have followed the suggestion that the non-perturbative biadjoint monopole of ref. [87] may be related to the singular Wu-Yang monopole solution in $\mathrm{SU}(2)$ gauge theory. There is no existing guidance on how to proceed, given that in all previous examples of the double copy, solutions of the linearised biadjoint field equation play a crucial role. For amplitudes, these are the denominator factors that act as scalar propagators. For the Kerr-Schild double copy, one relies upon a harmonic function $\phi$, that remains untouched (up to replacements of charge and mass factors) when moving between theories.

Our starting point was to note that shockwaves are known to double-copy, and that one can construct such solutions by ultraboosting static objects, by analogy with the seminal gravity study of ref. [91]. Furthermore, ultraboosting a solution results in a somewhat simpler structure, which might make it easier to spot how a nonperturbative double copy might work. To this end, we first showed how the Aichelburg-Sexl procedure, for the point charge and mass solutions considered in the original classical double copy of ref. [48], can be expressed in a form that makes the double copy manifest. This exercise was useful in itself: the ultraboost procedure has not been previously considered in biadjoint theory, and there are interesting aspects of how the double copy relates the ultraboosts in different theories (e.g. the need to rescale the charge parameters differently). Returning to the biadjoint monopole, we found that this effectively vanished upon performing an ultraboost, making any attempt to match it up with a gauge theory solution difficult.

We then explained the above observations by noting that the Wu-Yang monopole is related to a trivially dressed Dirac monopole by a (singular) gauge transformation. The latter, as implied by the study of ref. [49], maps to the well-known NUT solution in gravity. Consequently, upon ultraboosting the transformed Wu-Yang monopole, one may double copy the result to obtain the so-called NUT wave of ref. [97], which we verified by explicit calculation. Interestingly, we expressed the Dirac monopole in a gauge that was not in the right form for the usual Kerr-Schild double copy (e.g. the field was not null). However, the shockwaves we obtained were indeed of Kerr-Schild form, which may possibly be due to the highly constrained symmetry of the shockwave solutions. In addition, this then allowed us to take the zeroth copy of the boosted Dirac monopole, which is non-zero and thus not relatable to the biadjoint monopoles of ref. [87]. 
Our results provide a number of insights at the frontier of understanding of the remit of the double copy. Most significantly, we have uncovered the first example of an exact non-abelian solution - the Wu-Yang monopole - that can be identified with the same gravity solution as an abelian-like counterpart. This is analogous to similar behaviour observed in the study of scattering amplitudes [27, 36, 37], and indeed suggests that many more such examples can be found. This may provide clues about how to double copy exact non-abelian solutions without having to rely on the Kerr-Schild procedure, which would in turn greatly increase our understanding of the fundamental origin of the double copy itself.

\section{Acknowledgments}

We thank Donal O'Connell for a discussion, some time ago, about ultraboosting classical solutions, and David Berman for further useful discussions. NBA and RSM are supported by PhD studentships from the United Kingdom Science and Technology Facilities Council (STFC) and the Royal Society respectively. This work has been supported by the STFC Consolidated Grant ST/P000754/1 "String theory, gauge theory and duality", and by the European Union Horizon 2020 research and innovation programme under the Marie Skłodowska-Curie grant agreement No. 764850 "SAGEX".

\section{A Impulse calculations}

\section{A.1 Biadjoint impulse}

The impulse of a test particle of mass $m$ interacting with the ultraboosted biadjoint scalar field is related to the equations of motion for the interacting particle coupled with the biadjoint scalar [61],

$$
\frac{\partial p_{\mu}}{\partial t}=-\frac{\lambda}{m} c_{2}^{a} \tilde{c}_{2}^{a^{\prime}} \partial_{\mu} \Phi^{a a^{\prime}}
$$

Here, the biadjoint field $\Phi^{a \dot{a}}$ is given by the shockwave solution given by (3.19). Stripping out charge and mass parameters for the sake of simplicity, we have

$$
\frac{\partial p_{\mu}}{\partial t}=\partial_{\mu}\left(\ln \left(\frac{\rho^{2}}{\rho_{0}^{2}}\right) \delta(x-t)\right)
$$

Switching to lightcone coordinates $u=t-x, v=t+x$, the impulse experienced by the particle is

$$
\delta p_{\mu}=\int_{-\infty}^{\infty} d t \frac{\partial p_{\mu}}{\partial t}=\int_{-\infty}^{\infty} d u \frac{\partial p_{\mu}}{\partial u}=\int_{-\infty}^{\infty} d u \partial_{\mu}\left(\ln \left(\frac{\rho^{2}}{\rho_{0}^{2}}\right) \delta(u)\right)
$$

We will work this out for each component,

$$
\begin{aligned}
& \delta p_{u}=\int_{-\infty}^{\infty} d u \partial_{u}\left(\ln \left(\frac{\rho^{2}}{\rho_{0}^{2}}\right) \delta(u)\right)=\left.\ln \left(\frac{\rho^{2}}{\rho_{0}^{2}}\right) \delta(u)\right|_{u=-\infty} ^{\infty}=0 \\
& \delta p_{v}=0, \quad \delta p_{y}=\int_{-\infty}^{\infty} d u \frac{2 y}{\rho^{2}} \delta(u)=\frac{2 y}{\rho^{2}}, \quad \delta p_{z}=\int_{-\infty}^{\infty} d u \frac{2 z}{\rho^{2}} \delta(u)=\frac{2 z}{\rho^{2}}
\end{aligned}
$$


Altogether, the interacting particle experiences an impulse with charges and couplings reinstated:

$$
\delta p^{\mu}=-\frac{\lambda^{2}}{m} \frac{c \cdot c_{2} \tilde{c} \cdot \tilde{c}_{2}}{2 \pi \rho^{2}}(0,0, y, z) .
$$

\section{A.2 Gauge theory impulse}

The impulse of a particle interacting with the ultraboosted gauge field is related to the Lorentz force, given by

$$
\frac{\partial p^{\mu}}{\partial t}=g c_{2}^{a} F_{\nu}^{a \mu} v^{\nu}
$$

As we are working in the rest frame of the interacting particle, we only need to consider the following components of the field strength tensor:

$$
F_{0}^{a \mu}=\partial^{\mu} A_{0}^{a}-\partial_{0} A^{a \mu}
$$

where $A^{\mu}$ is the shockwave gauge potential given by the ultraboosted field given by (3.17), as well as $A_{\mu}=A_{\mu}^{a} T^{a}$, and $\partial^{\mu}=\left(\partial_{t},-\partial_{x},-\partial_{y},-\partial_{z}\right)$. The components of interest of the field strength tensor are

$$
\begin{aligned}
& F_{0}^{a 1}=\frac{g c^{a}}{4 \pi} \ln \left(\frac{\rho^{2}}{\rho_{0}^{2}}\right)\left(\partial_{t}+\partial_{x}\right) \delta(x-t)=0 \\
& F_{0}^{a j}=\frac{g c^{a}}{4 \pi} \delta(x-t) \partial_{j} \ln \left(\frac{\rho^{2}}{\rho_{0}^{2}}\right)=\frac{c^{a}}{4 \pi} \delta(x-t) \frac{2 x^{j}}{\rho^{2}}
\end{aligned}
$$

where $j=2,3$ or equivalently $x^{j}=y, z$. We can now extract the impulse,

$$
\delta p^{\mu}=\int_{-\infty}^{\infty} d t \frac{d p^{\mu}}{d t} \rightarrow \delta p^{j}=\int_{-\infty}^{\infty} d t \frac{c_{2} \cdot c}{4 \pi} \delta(x-t) \frac{2 x^{j}}{\rho^{2}}=\frac{c_{2} \cdot c}{4 \pi} \frac{2 x^{j}}{\rho^{2}}
$$

which leaves us finally with the impulse

$$
\delta p^{\mu}: \frac{g^{2} c_{2} \cdot c}{2 \pi \rho^{2}}(0,0, y, z)
$$

This is identical (setting aside couplings and charges) to the biadjoint case. If the interacting particle were to have the same charge as that producing the shockwave, the effect of the impulse would be to send the particle away from the shockwave nucleus. Opposite charges would result in the particle being drawn in closer to the nucleus. The strength of the push or pull depends on how far the particle is initially from the source of the shockwave. The closer the initial separation, the stronger the impulse.

\section{A.3 Gravitational impulse}

The impulse of a particle of mass $m$ interacting with the ultraboosted graviton field is related to the geodesic equation given by

$$
\frac{\partial p^{\mu}}{\partial t}=-m \Gamma_{\nu \sigma}^{\mu} v^{\nu} v^{\sigma}
$$


The metric associated with shockwave geometry is

$$
g_{\mu \nu}=\eta_{\mu \nu}+\frac{\kappa^{2}}{2} \frac{M}{4 \pi} \delta(x-t) \ln \left(\frac{\rho^{2}}{\rho_{0}^{2}}\right) \bar{k}_{\mu} \bar{k}_{\nu}
$$

with inverse

$$
g^{\mu \nu}=\eta^{\mu \nu}-\frac{\kappa^{2}}{2} \frac{M}{4 \pi} \delta(x-t) \ln \left(\frac{\rho^{2}}{\rho_{0}^{2}}\right) \bar{k}^{\mu} \bar{k}^{\nu} .
$$

As we are in the rest frame of the particle interacting with our shockwave, the geodesic equation simplifies greatly to

$$
\frac{\partial p^{\mu}}{\partial t}=-m \Gamma_{00}^{\mu}
$$

We only need 4 Christoffel symbols:

$$
\begin{aligned}
\Gamma_{00}^{0} & =\frac{-1}{2} \frac{\kappa^{2}}{2} \frac{M}{4 \pi} \ln \left(\frac{\rho^{2}}{\rho_{0}^{2}}\right) \partial_{t} \delta(x-t), & \Gamma_{00}^{x} & =\frac{1}{2} \frac{\kappa^{2}}{2} \frac{M}{4 \pi} \ln \left(\frac{\rho^{2}}{\rho_{0}^{2}}\right) \partial_{t} \delta(x-t), \\
\Gamma_{00}^{y} & =\frac{\kappa^{2}}{2} \frac{M}{4 \pi} \frac{y}{\rho^{2}} \delta(x-t), & \Gamma_{00}^{z} & =\frac{\kappa^{2}}{2} \frac{M}{4 \pi} \frac{z}{\rho^{2}} \delta(x-t) .
\end{aligned}
$$

The impulse $\delta p^{\mu}$ is then the time integral of these Christoffel symbols,

$$
\begin{aligned}
\delta p^{0}=-\delta p^{x} & =\frac{\kappa^{2}}{2} \frac{M m}{4 \pi} \int_{-\infty}^{\infty} d t \frac{1}{2} \ln \left(\frac{\rho^{2}}{\rho_{0}^{2}}\right) \partial_{t} \delta(x-t) \\
& =\frac{\kappa^{2}}{4} \frac{M m}{4 \pi} \ln \left(\frac{\rho^{2}}{\rho_{0}^{2}}\right) \int_{-\infty}^{\infty} d u \partial_{u} \delta(u) \\
& =\left.\frac{\kappa^{2}}{4} \frac{M m}{4 \pi} \ln \left(\frac{\rho^{2}}{\rho_{0}^{2}}\right) \delta(u)\right|_{u=-\infty} ^{\infty}=0 \\
\delta p^{y} & =\frac{-\kappa^{2}}{2} \frac{M m}{4 \pi} \int_{-\infty}^{\infty} d t \delta(x-t) \frac{y}{\rho^{2}}=\frac{-\kappa^{2}}{2} \frac{M m}{4 \pi} \frac{y}{\rho^{2}} \\
\delta p^{z} & =\frac{-\kappa^{2}}{2} \frac{M m}{4 \pi} \int_{-\infty}^{\infty} d t \delta(x-t) \frac{z}{\rho^{2}}=\frac{-\kappa^{2}}{2} \frac{M m}{4 \pi} \frac{z}{\rho^{2}}
\end{aligned}
$$

The impulse bears a resemblance to those of the biadjoint scalar and gauge theories, i.e.

$$
\delta p^{\mu}=\frac{-\kappa^{2} M m}{8 \pi \rho^{2}}(0,0, y, z)
$$

The particle gets a kick restricted to the $y-z$ plane, with the magnitude of the kick diminishing the further the particle is from the nucleus of the shockwave. We would expect, as gravity is an attractive force, that the impulse should draw the particle toward the nucleus of the shockwave. By contrast, the gauge theory case is repulsive (provided $c_{2} \cdot c$ is positive in eq. (A.9)), which results in the sign difference between eq. (A.9) and eq. (A.15). 


\section{B Ultraboosting the general $\mathrm{SU}(2) \times \mathrm{SU}(2)$ monopole}

In section 3.3, we ultraboosted the biadjoint monopole solution of eq. (2.15), finding that it disappears in the ultrarelativistic limit. In this appendix, we show that a similar conclusion is reached for the more general monopole solutions of eq. (2.17), that were are also derived in ref. [87]. Recall that these solutions have the form

$$
\Phi^{a a^{\prime}}=\frac{1}{\lambda r^{2}}\left[-k\left(\delta^{a a^{\prime}}-\frac{x^{a} x^{a^{\prime}}}{r^{2}}\right) \pm \sqrt{2 k-k^{2}} \frac{\epsilon^{a a^{\prime} d} x^{d}}{r}\right], \quad 0 \leq k \leq 2 .
$$

Intermediate details of the ultraboost calculation are cumbersome due to selecting a particular boost direction, so we will simply quote the results. As in section 3.3, we boost in the $x$-direction, and examine the behaviour of the boosted solution inside and outside the plane $x=\beta t$. Outside of the plane, we find that the solution vanishes completely:

$$
\Phi^{a a^{\prime}} \stackrel{\gamma \rightarrow \infty}{\longrightarrow} 0 \quad \text { for } x-\beta t \neq 0 .
$$

Inside of the plane, just as for the ultraboost of the (3.30), the field takes finite values and displays no divergent behaviour. More specifically, in the limit $\gamma \rightarrow \infty$ we find

$$
\begin{aligned}
& \Phi^{11} \rightarrow \frac{-k}{\lambda\left(y^{2}+z^{2}\right)}, \\
& \Phi^{22} \rightarrow \frac{-k z^{2}}{\lambda\left(y^{2}+z^{2}\right)^{2}}, \\
& \Phi^{33} \rightarrow \frac{-k y^{2}}{\lambda\left(y^{2}+z^{2}\right)^{2}}, \\
& \Phi^{12}=-\Phi^{21} \rightarrow \frac{z \sqrt{\left(k^{2}-2 k\right)}}{\lambda\left(y^{2}+z^{2}\right)^{3 / 2}}, \quad \Phi^{13}=-\Phi^{31} \rightarrow-\frac{y \sqrt{\left(k^{2}-2 k\right)}}{\lambda\left(y^{2}+z^{2}\right)^{3 / 2}}, \quad \Phi^{23}=\Phi^{32} \rightarrow \frac{y z k}{\lambda\left(y^{2}+z^{2}\right)^{2}} .
\end{aligned}
$$

We thus conclude that, similarly to (3.30), the solutions of eq. (2.17) remain finite in the plane $x=\beta t$ after the ultraboost (i.e. do not produce a delta function). Thus, they do not constitute shockwaves. Similarly to (2.15), one can produce a shockwave solution by rescaling the coupling according to $\lambda \rightarrow \lambda / \gamma$. As mentioned below (3.31) however, this entails changing the theory, and is not analogous to the Aichelburg-Sexl-like rescalings, which affect the charges of the objects being boosted.

Open Access. This article is distributed under the terms of the Creative Commons Attribution License (CC-BY 4.0), which permits any use, distribution and reproduction in any medium, provided the original author(s) and source are credited.

\section{References}

[1] Z. Bern, J.J.M. Carrasco and H. Johansson, New Relations for Gauge-Theory Amplitudes, Phys. Rev. D 78 (2008) 085011 [arXiv:0805.3993] [InSPIRE].

[2] Z. Bern, J.J.M. Carrasco and H. Johansson, Perturbative Quantum Gravity as a Double Copy of Gauge Theory, Phys. Rev. Lett. 105 (2010) 061602 [arXiv:1004.0476] [InSPIRE].

[3] Z. Bern, T. Dennen, Y.-t. Huang and M. Kiermaier, Gravity as the Square of Gauge Theory, Phys. Rev. D 82 (2010) 065003 [arXiv: 1004.0693] [InSPIRE].

[4] Z. Bern, L.J. Dixon, D.C. Dunbar, M. Perelstein and J.S. Rozowsky, On the relationship between Yang-Mills theory and gravity and its implication for ultraviolet divergences, Nucl. Phys. B 530 (1998) 401 [hep-th/9802162] [INSPIRE]. 
[5] M.B. Green, J.H. Schwarz and L. Brink, $N=4$ Yang-Mills and $N=8$ Supergravity as Limits of String Theories, Nucl. Phys. B 198 (1982) 474 [InSPIRE].

[6] Z. Bern, J.S. Rozowsky and B. Yan, Two loop four gluon amplitudes in $N=4$ superYang-Mills, Phys. Lett. B 401 (1997) 273 [hep-ph/9702424] [INSPIRE].

[7] J.J.M. Carrasco and H. Johansson, Five-Point Amplitudes in $N=4$ Super-Yang-Mills Theory and $N=8$ Supergravity, Phys. Rev. D 85 (2012) 025006 [arXiv:1106.4711] [INSPIRE].

[8] J.J.M. Carrasco, M. Chiodaroli, M. Günaydin and R. Roiban, One-loop four-point amplitudes in pure and matter-coupled $N \leq 4$ supergravity, JHEP 03 (2013) 056 [arXiv:1212.1146] [INSPIRE].

[9] C.R. Mafra and O. Schlotterer, The Structure of n-Point One-Loop Open Superstring Amplitudes, JHEP 08 (2014) 099 [arXiv:1203.6215] [INSPIRE].

[10] R.H. Boels, R.S. Isermann, R. Monteiro and D. O'Connell, Colour-Kinematics Duality for One-Loop Rational Amplitudes, JHEP 04 (2013) 107 [arXiv:1301.4165] [INSPIRE].

[11] N.E.J. Bjerrum-Bohr, T. Dennen, R. Monteiro and D. O'Connell, Integrand Oxidation and One-Loop Colour-Dual Numerators in N=4 Gauge Theory, JHEP 07 (2013) 092 [arXiv:1303.2913] [INSPIRE].

[12] Z. Bern, S. Davies, T. Dennen, Y.-t. Huang and J. Nohle, Color-Kinematics Duality for Pure Yang-Mills and Gravity at One and Two Loops, Phys. Rev. D 92 (2015) 045041 [arXiv: 1303.6605] [INSPIRE].

[13] Z. Bern, S. Davies and T. Dennen, The Ultraviolet Structure of Half-Maximal Supergravity with Matter Multiplets at Two and Three Loops, Phys. Rev. D 88 (2013) 065007 [arXiv: 1305.4876] [INSPIRE].

[14] J. Nohle, Color-Kinematics Duality in One-Loop Four-Gluon Amplitudes with Matter, Phys. Rev. D 90 (2014) 025020 [arXiv:1309.7416] [INSPIRE].

[15] Z. Bern, S. Davies, T. Dennen, A.V. Smirnov and V.A. Smirnov, Ultraviolet Properties of $N=4$ Supergravity at Four Loops, Phys. Rev. Lett. 111 (2013) 231302 [arXiv:1309.2498] [INSPIRE].

[16] S.G. Naculich, H. Nastase and H.J. Schnitzer, All-loop infrared-divergent behavior of most-subleading-color gauge-theory amplitudes, JHEP 04 (2013) 114 [arXiv:1301.2234] [INSPIRE].

[17] Y.-J. Du, B. Feng and C.-H. Fu, Dual-color decompositions at one-loop level in Yang-Mills theory, JHEP 06 (2014) 157 [arXiv: 1402.6805] [INSPIRE].

[18] C.R. Mafra and O. Schlotterer, Towards one-loop SYM amplitudes from the pure spinor BRST cohomology, Fortsch. Phys. 63 (2015) 105 [arXiv: 1410.0668] [inSPIRE].

[19] Z. Bern, S. Davies and T. Dennen, Enhanced ultraviolet cancellations in $\mathcal{N}=5$ supergravity at four loops, Phys. Rev. D 90 (2014) 105011 [arXiv:1409.3089] [INSPIRE].

[20] C.R. Mafra and O. Schlotterer, Two-loop five-point amplitudes of super Yang-Mills and supergravity in pure spinor superspace, JHEP 10 (2015) 124 [arXiv:1505. 02746] [INSPIRE].

[21] S. He, R. Monteiro and O. Schlotterer, String-inspired BCJ numerators for one-loop MHV amplitudes, JHEP 01 (2016) 171 [arXiv:1507.06288] [INSPIRE].

[22] Z. Bern, S. Davies and J. Nohle, Double-Copy Constructions and Unitarity Cuts, Phys. Rev. D 93 (2016) 105015 [arXiv:1510.03448] [INSPIRE]. 
[23] G. Mogull and D. O'Connell, Overcoming Obstacles to Colour-Kinematics Duality at Two Loops, JHEP 12 (2015) 135 [arXiv:1511.06652] [INSPIRE].

[24] M. Chiodaroli, M. Günaydin, H. Johansson and R. Roiban, Spontaneously Broken Yang-Mills-Einstein Supergravities as Double Copies, JHEP 06 (2017) 064 [arXiv: 1511.01740] [INSPIRE].

[25] Z. Bern, J.J.M. Carrasco, W.-M. Chen, H. Johansson, R. Roiban and M. Zeng, Five-loop four-point integrand of $N=8$ supergravity as a generalized double copy, Phys. Rev. D 96 (2017) 126012 [arXiv:1708.06807] [inSPIRE].

[26] H. Johansson and A. Ochirov, Color-Kinematics Duality for QCD Amplitudes, JHEP 01 (2016) 170 [arXiv: 1507.00332] [INSPIRE].

[27] S. Oxburgh and C.D. White, BCJ duality and the double copy in the soft limit, JHEP 02 (2013) 127 [arXiv:1210.1110] [INSPIRE].

[28] Y. Geyer, R. Monteiro and R. Stark-Muchão, Two-Loop Scattering Amplitudes: Double-Forward Limit and Colour-Kinematics Duality, JHEP 12 (2019) 049 [arXiv: 1908.05221] [INSPIRE].

[29] C.D. White, Factorization Properties of Soft Graviton Amplitudes, JHEP 05 (2011) 060 [arXiv:1103.2981] [INSPIRE].

[30] S. Melville, S.G. Naculich, H.J. Schnitzer and C.D. White, Wilson line approach to gravity in the high energy limit, Phys. Rev. D 89 (2014) 025009 [arXiv:1306.6019] [INSPIRE].

[31] A. Luna, S. Melville, S.G. Naculich and C.D. White, Next-to-soft corrections to high energy scattering in QCD and gravity, JHEP 01 (2017) 052 [arXiv: 1611.02172] [INSPIRE].

[32] R. Saotome and R. Akhoury, Relationship Between Gravity and Gauge Scattering in the High Energy Limit, JHEP 01 (2013) 123 [arXiv:1210.8111] [INSPIRE].

[33] A. Sabio Vera, E. Serna Campillo and M.A. Vazquez-Mozo, Color-Kinematics Duality and the Regge Limit of Inelastic Amplitudes, JHEP 04 (2013) 086 [arXiv:1212.5103] [INSPIRE].

[34] H. Johansson, A. Sabio Vera, E. Serna Campillo and M.Á. Vázquez-Mozo, Color-Kinematics Duality in Multi-Regge Kinematics and Dimensional Reduction, JHEP 10 (2013) 215 [arXiv: 1307.3106] [INSPIRE].

[35] H. Johansson, A. Sabio Vera, E. Serna Campillo and M.A. Vazquez-Mozo, Color-kinematics duality and dimensional reduction for graviton emission in Regge limit, in International Workshop on Low X Physics (Israel 2013), Eilat, Israel, 30 May-4 June 2013 (2013) [arXiv:1310.1680] [INSPIRE].

[36] T. Bargheer, S. He and T. McLoughlin, New Relations for Three-Dimensional Supersymmetric Scattering Amplitudes, Phys. Rev. Lett. 108 (2012) 231601 [arXiv: 1203.0562] [INSPIRE].

[37] Y.-t. Huang and H. Johansson, Equivalent D $=3$ Supergravity Amplitudes from Double Copies of Three-Algebra and Two-Algebra Gauge Theories, Phys. Rev. Lett. 110 (2013) 171601 [arXiv: 1210.2255] [INSPIRE].

[38] G. Chen and Y.-J. Du, Amplitude Relations in Non-linear $\sigma$-model, JHEP 01 (2014) 061 [arXiv: 1311.1133] [INSPIRE].

[39] M. Chiodaroli, Q. Jin and R. Roiban, Color/kinematics duality for general abelian orbifolds of $N=4$ super Yang-Mills theory, JHEP 01 (2014) 152 [arXiv:1311.3600] [INSPIRE]. 
[40] H. Johansson and A. Ochirov, Pure Gravities via Color-Kinematics Duality for Fundamental Matter, JHEP 11 (2015) 046 [arXiv: 1407.4772] [INSPIRE].

[41] H. Johansson and J. Nohle, Conformal Gravity from Gauge Theory, arXiv:1707.02965 [INSPIRE].

[42] M. Chiodaroli, M. Günaydin, H. Johansson and R. Roiban, Gauged Supergravities and Spontaneous Supersymmetry Breaking from the Double Copy Construction, Phys. Rev. Lett. 120 (2018) 171601 [arXiv:1710.08796] [INSPIRE].

[43] G. Chen, H. Johansson, F. Teng and T. Wang, On the kinematic algebra for BCJ numerators beyond the MHV sector, JHEP 11 (2019) 055 [arXiv: 1906.10683] [INSPIRE].

[44] J. Plefka, C. Shi and T. Wang, Double copy of massive scalar QCD, Phys. Rev. D 101 (2020) 066004 [arXiv: 1911.06785] [inSPIRE].

[45] R. Aoude and A. Helset, Soft Theorems and the KLT-Relation, JHEP 04 (2020) 044 [arXiv: 1911.09962] [INSPIRE].

[46] A.E. Lipstein and P. McFadden, Double copy structure and the flat space limit of conformal correlators in even dimensions, arXiv:1912.10046 [INSPIRE].

[47] Z. Bern, J.J. Carrasco, M. Chiodaroli, H. Johansson and R. Roiban, The Duality Between Color and Kinematics and its Applications, arXiv:1909.01358 [INSPIRE].

[48] R. Monteiro, D. O'Connell and C.D. White, Black holes and the double copy, JHEP 12 (2014) 056 [arXiv: 1410.0239] [inSPIRE].

[49] A. Luna, R. Monteiro, D. O'Connell and C.D. White, The classical double copy for Taub-NUT spacetime, Phys. Lett. B 750 (2015) 272 [arXiv:1507.01869] [InSPIRE].

[50] A. Luna, R. Monteiro, I. Nicholson, D. O'Connell and C.D. White, The double copy: Bremsstrahlung and accelerating black holes, JHEP 06 (2016) 023 [arXiv: 1603.05737] [INSPIRE].

[51] W.D. Goldberger and A.K. Ridgway, Radiation and the classical double copy for color charges, Phys. Rev. D 95 (2017) 125010 [arXiv:1611.03493] [INSPIRE].

[52] A. Anastasiou, L. Borsten, M.J. Duff, L.J. Hughes and S. Nagy, Yang-Mills origin of gravitational symmetries, Phys. Rev. Lett. 113 (2014) 231606 [arXiv:1408.4434] [INSPIRE].

[53] L. Borsten and M.J. Duff, Gravity as the square of Yang-Mills?, Phys. Scripta 90 (2015) 108012 [arXiv: 1602 .08267] [INSPIRE].

[54] A. Anastasiou et al., Twin supergravities from Yang-Mills theory squared, Phys. Rev. D 96 (2017) 026013 [arXiv: 1610.07192] [inSPIRE].

[55] A. Anastasiou, L. Borsten, M.J. Duff, A. Marrani, S. Nagy and M. Zoccali, Are all supergravity theories Yang-Mills squared?, Nucl. Phys. B 934 (2018) 606 [arXiv: 1707.03234] [INSPIRE].

[56] G.L. Cardoso, S. Nagy and S. Nampuri, A double copy for $\mathcal{N}=2$ supergravity: a linearised tale told on-shell, JHEP 10 (2016) 127 [arXiv:1609.05022] [INSPIRE].

[57] L. Borsten, $D=6, \mathcal{N}=(2,0)$ and $\mathcal{N}=(4,0)$ theories, Phys. Rev. D 97 (2018) 066014 [arXiv: 1708.02573] [INSPIRE].

[58] A. Anastasiou, L. Borsten, M.J. Duff, A. Marrani, S. Nagy and M. Zoccali, The Mile High Magic Pyramid, arXiv:1711.08476 [INSPIRE]. 
[59] A. Anastasiou, L. Borsten, M.J. Duff, S. Nagy and M. Zoccali, Gravity as Gauge Theory Squared: A Ghost Story, Phys. Rev. Lett. 121 (2018) 211601 [arXiv:1807.02486] [InSPIRE].

[60] G. Lopes Cardoso, G. Inverso, S. Nagy and S. Nampuri, Comments on the double copy construction for gravitational theories, PoS (CORFU2017)177 (2018) [arXiv:1803.07670] [INSPIRE].

[61] W.D. Goldberger, S.G. Prabhu and J.O. Thompson, Classical gluon and graviton radiation from the bi-adjoint scalar double copy, Phys. Rev. D 96 (2017) 065009 [arXiv:1705.09263] [INSPIRE].

[62] W.D. Goldberger and A.K. Ridgway, Bound states and the classical double copy, Phys. Rev. D 97 (2018) 085019 [arXiv:1711.09493] [INSPIRE].

[63] W.D. Goldberger, J. Li and S.G. Prabhu, Spinning particles, axion radiation and the classical double copy, Phys. Rev. D 97 (2018) 105018 [arXiv:1712.09250] [INSPIRE].

[64] A. Luna et al., Perturbative spacetimes from Yang-Mills theory, JHEP 04 (2017) 069 [arXiv: 1611.07508] [INSPIRE].

[65] A. Luna, I. Nicholson, D. O'Connell and C.D. White, Inelastic Black Hole Scattering from Charged Scalar Amplitudes, JHEP 03 (2018) 044 [arXiv:1711.03901] [INSPIRE].

[66] C.-H. Shen, Gravitational Radiation from Color-Kinematics Duality, JHEP 11 (2018) 162 [arXiv: 1806. 07388] [INSPIRE].

[67] M. Levi, Effective Field Theories of Post-Newtonian Gravity: A comprehensive review, arXiv: 1807.01699 [INSPIRE].

[68] R.A. Porto, The effective field theorist's approach to gravitational dynamics, Phys. Rept. 633 (2016) 1 [arXiv: 1601.04914] [INSPIRE].

[69] J. Plefka, J. Steinhoff and W. Wormsbecher, Effective action of dilaton gravity as the classical double copy of Yang-Mills theory, Phys. Rev. D 99 (2019) 024021 [arXiv:1807.09859] [INSPIRE].

[70] C. Cheung, I.Z. Rothstein and M.P. Solon, From Scattering Amplitudes to Classical Potentials in the Post-Minkowskian Expansion, Phys. Rev. Lett. 121 (2018) 251101 [arXiv: 1808.02489] [INSPIRE].

[71] M. Carrillo González, R. Penco and M. Trodden, Radiation of scalar modes and the classical double copy, JHEP 11 (2018) 065 [arXiv: 1809.04611] [INSPIRE].

[72] R. Monteiro, I. Nicholson and D. O'Connell, Spinor-helicity and the algebraic classification of higher-dimensional spacetimes, Class. Quant. Grav. 36 (2019) 065006 [arXiv: 1809.03906] [INSPIRE].

[73] J. Plefka, C. Shi, J. Steinhoff and T. Wang, Breakdown of the classical double copy for the effective action of dilaton-gravity at NNLO, Phys. Rev. D 100 (2019) 086006 [arXiv: 1906.05875] [INSPIRE].

[74] B. Maybee, D. O'Connell and J. Vines, Observables and amplitudes for spinning particles and black holes, JHEP 12 (2019) 156 [arXiv:1906.09260] [INSPIRE].

[75] H. Johansson and A. Ochirov, Double copy for massive quantum particles with spin, JHEP 09 (2019) 040 [arXiv: 1906.12292] [INSPIRE].

[76] A.P.V. and A. Manu, Classical double copy from Color Kinematics duality: A proof in the soft limit, Phys. Rev. D 101 (2020) 046014 [arXiv:1907.10021] [INSPIRE]. 
[77] M. Carrillo González, R. Penco and M. Trodden, Shift symmetries, soft limits and the double copy beyond leading order, arXiv:1908.07531 [INSPIRE].

[78] Y.F. Bautista and A. Guevara, On the Double Copy for Spinning Matter, arXiv: 1908.11349 [INSPIRE].

[79] N. Moynihan, Kerr-Newman from Minimal Coupling, JHEP 01 (2020) 014 [arXiv: 1909.05217] [INSPIRE].

[80] I. Bah, R. Dempsey and P. Weck, Kerr-Schild Double Copy and Complex Worldlines, JHEP 02 (2020) 180 [arXiv: 1910.04197] [inSPIRE].

[81] M. Carrillo González, B. Melcher, K. Ratliff, S. Watson and C.D. White, The classical double copy in three spacetime dimensions, JHEP 07 (2019) 167 [arXiv:1904.11001] [INSPIRE].

[82] W.D. Goldberger and J. Li, Strings, extended objects and the classical double copy, JHEP 02 (2020) 092 [arXiv: 1912.01650] [INSPIRE].

[83] K. Kim, K. Lee, R. Monteiro, I. Nicholson and D. Peinador Veiga, The Classical Double Copy of a Point Charge, JHEP 02 (2020) 046 [arXiv: 1912.02177] [INSPIRE].

[84] A. Banerjee, E. Ó Colgáin, J.A. Rosabal and H. Yavartanoo, Ehlers as EM duality in the double copy, arXiv:1912.02597 [INSPIRE].

[85] A. Ilderton, Screw-symmetric gravitational waves: a double copy of the vortex, Phys. Lett. B $782(2018) 22$ [arXiv: 1804.07290] [INSPIRE].

[86] A. Luna, R. Monteiro, I. Nicholson and D. O'Connell, Type D Spacetimes and the Weyl Double Copy, Class. Quant. Grav. 36 (2019) 065003 [arXiv:1810.08183] [INSPIRE].

[87] C.D. White, Exact solutions for the biadjoint scalar field, Phys. Lett. B 763 (2016) 365 [arXiv: 1606.04724] [INSPIRE].

[88] P.-J. De Smet and C.D. White, Extended solutions for the biadjoint scalar field, Phys. Lett. B 775 (2017) 163 [arXiv: 1708.01103] [INSPIRE].

[89] N. Bahjat-Abbas, R. Stark-Muchão and C.D. White, Biadjoint wires, Phys. Lett. B 788 (2019) 274 [arXiv: 1810.08118] [INSPIRE].

[90] T.T. Wu and C.-N. Yang, Some solutions of the classical isotopic gauge field equations, in Properties of Matter Under Unusual Conditions, (1967) [INSPIRE].

[91] P.C. Aichelburg and R.U. Sexl, On the Gravitational field of a massless particle, Gen. Rel. Grav. 2 (1971) 303 [InSPIRE].

[92] C.O. Lousto and N.G. Sanchez, The Curved Shock Wave Space-time of Ultrarelativistic Charged Particles and Their Scattering, Int. J. Mod. Phys. A 5 (1990) 915 [inSPIRE].

[93] C.O. Lousto and N.G. Sanchez, Gravitational Shock Waves of Ultrahigh Energetic Particles on Curved Space-times, Phys. Lett. B 220 (1989) 55 [INSPIRE].

[94] C.O. Lousto and N.G. Sanchez, The Ultrarelativistic Limit of the Kerr-Newman Geometry and Particle Scattering at the Planck Scale, Phys. Lett. B 232 (1989) 462 [InSPIRE].

[95] C.O. Lousto and N.G. Sanchez, The Ultrarelativistic limit of the boosted Kerr-Newman geometry and the scattering of spin 1/2 particles, Nucl. Phys. B 383 (1992) 377 [INSPIRE].

[96] M.V. Kozyulin and Z.K. Silagadze, Light bending by a Coulomb field and the Aichelburg-Sexl ultraboost, Eur. J. Phys. 32 (2011) 1357 [arXiv:1104.2557] [InSPIRE]. 
[97] R. Argurio, F. Dehouck and L. Houart, Boosting Taub-NUT to a BPS NUT-wave, JHEP 01 (2009) 045 [arXiv:0811.0538] [INSPIRE].

[98] W. Siegel, Fields, hep-th/9912205 [INSPIRE].

[99] R.A. Brandt and F. Neri, Stability Analysis for Singular Nonabelian Magnetic Monopoles, Nucl. Phys. B 161 (1979) 253 [InSPIRE].

[100] R.A. Brandt and F. Neri, Magnetic Monopoles in SU(N) Gauge Theories, Nucl. Phys. B 186 (1981) 84 [INSPIRE].

[101] J.J.M. Carrasco, Gauge and Gravity Amplitude Relations, in Proceedings, Theoretical Advanced Study Institute in Elementary Particle Physics: Journeys Through the Precision Frontier: Amplitudes for Colliders (TASI 2014), Boulder, Colorado, 2-27 June 2014, pp. 477-557, WSP (2015) [DOI] [arXiv:1506.00974] [INSPIRE].

[102] C. Cheung, C.-H. Shen and C. Wen, Unifying Relations for Scattering Amplitudes, JHEP 02 (2018) 095 [arXiv: 1705.03025] [INSPIRE].

[103] J.J.M. Carrasco and L. Rodina, UV considerations on scattering amplitudes in a web of theories, Phys. Rev. D 100 (2019) 125007 [arXiv:1908. 08033] [INSPIRE].

[104] N. Bahjat-Abbas, A. Luna and C.D. White, The Kerr-Schild double copy in curved spacetime, JHEP 12 (2017) 004 [arXiv:1710.01953] [INSPIRE].

[105] D.S. Berman, E. Chacón, A. Luna and C.D. White, The self-dual classical double copy and the Eguchi-Hanson instanton, JHEP 01 (2019) 107 [arXiv: 1809.04063] [INSPIRE].

[106] A.K. Ridgway and M.B. Wise, Static Spherically Symmetric Kerr-Schild Metrics and Implications for the Classical Double Copy, Phys. Rev. D 94 (2016) 044023 [arXiv: 1512.02243] [INSPIRE].

[107] M. Carrillo-González, R. Penco and M. Trodden, The classical double copy in maximally symmetric spacetimes, JHEP 04 (2018) 028 [arXiv:1711.01296] [INSPIRE].

[108] S.R. Coleman, Nonabelian Plane Waves, Phys. Lett. 70B (1977) 59 [InSPIRE].

[109] Z.W. Chong, G.W. Gibbons, H. Lü and C.N. Pope, Separability and Killing tensors in Kerr-Taub-NUT-de Sitter metrics in higher dimensions, Phys. Lett. B 609 (2005) 124 [hep-th/0405061] [INSPIRE].

[110] Y.-T. Huang, U. Kol and D. O'Connell, The Double Copy of Electric-Magnetic Duality, arXiv: 1911.06318 [INSPIRE].

[111] R. Alawadhi, D. Peinador Veiga, D.S. Berman and B. Spence, S-duality and the double copy, JHEP 03 (2020) 059 [arXiv: 1911.06797] [INSPIRE].

[112] L. Borsten, I. Jubb, V. Makwana and S. Nagy, Gauge $\times$ Gauge on Spheres, arXiv:1911.12324 [INSPIRE].

[113] G. 't Hooft, Magnetic Monopoles in Unified Gauge Theories, Nucl. Phys. B 79 (1974) 276 [INSPIRE].

[114] A.M. Polyakov, Isomeric States of Quantum Fields, Zh. Eksp. Teor. Fiz. 68 (1975) 1975 [INSPIRE].

[115] P. Sikivie and N. Weiss, Classical Yang-Mills Theory in the Presence of External Sources, Phys. Rev. D 18 (1978) 3809 [InSPIRE]. 